\title{
Evaluation of the pancreatic tumors by transabdominal Shear Wave Elastography: preliminary results of a pilot study
}

\author{
Razvan Zaro', Dina Liliana ${ }^{2}$, Cristina Pojoga ${ }^{2}$, Stefan Vesa ${ }^{3}$, Radu Badea ${ }^{1}$ \\ ${ }^{1}$ Ultrasound Imaging Department, ${ }^{2}$ Gastroenterology Department, "Octavian Fodor" Regional Institute of Gastroen- \\ terology and Hepatology, ${ }^{3}$ Department of Pharmacology, Toxicology and Clinical Pharmacology, "Iuliu Hațieganu" \\ University of Medicine and Pharmacy, Cluj-Napoca, Romania
}

\begin{abstract}
Aim: To identify the mean values of Shear Wave Elastography (SWE) in pancreatic neoplasms. Material and method: This pilot case-control study was conducted in a tertiary care setting and data were collected in a prospective manner. Thirty-three subjects were included and divided into two groups: 18 healthy subjects and 15 patients with pancreatic neoplasm, from which 14 patients were diagnosed with adenocarcinoma and one with intraductal papillary mucinous neoplasia. Inclusion criteria for patients with pancreatic neoplasia were histopathological confirmation obtained by endoscopic ultrasonography (EUS) with fine needle aspiration or surgically and imaging confirmation by computed tomography with contrast enhancement. A number of minimum 5 shear wave velocity (SWV) measurements regarding each segment respectively at the tumoral level was obtained among a series of elastograms. Results: The mean SWV of the pancreas in the control group was $1.21 \pm 0.27 \mathrm{~m} / \mathrm{s}$. Data regarding the pathological parenchyma indicated an increase of the SWV at the tumoral (cephalic) level corresponding to $1.54 \pm 0.32 \mathrm{~m} / \mathrm{s}$, with significant statistical difference between the control batch and tumoral group, $\mathrm{p}=0.02$. Conclusions: Transabdominal SWE represents a surrogate but feasible method for differentiation cancer from the normal pancreatic tissue. In a clinical setting, data regarding the stiffness complementary to an ultrasound evaluation could guide high-risk patients for a close-up pancreatic monitoring
\end{abstract}

Keywords: transabdominal elastography; Shear Wave Elastography; pancreatic adenocarcinoma

\section{Introduction}

Pancreatic cancer shows an average 5-year survival rate of $5 \%$. Frequently, the tumors are found in a late stage, have invasive and aggressive behavior and complex imaging investigations are required for diagnosis. The only potentially curative treatment is surgical resection, but this applies to only $15-20 \%$ of patients [1] Clinically, asthenia, weight loss, upper abdominal pain (56-86\%), vesicular hydrops, hepatomegaly and jaundice are observed [2].

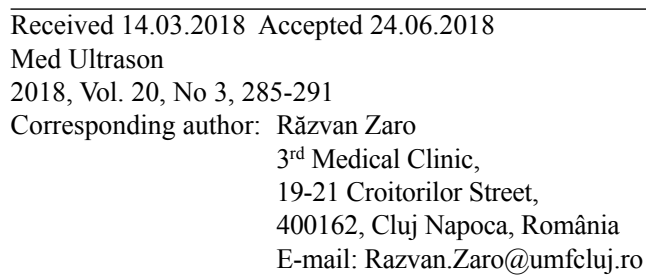

Pancreatic ductal adenocarcinoma (PDAC) accounts for approximately $95 \%$ [1] of pancreatic malignant tumors and over two-thirds are localized to the head and uncinate process. Pancreatic tumor lesions, either solid or cystic, benign or malignant, raise diagnostic and interdisciplinary treatment issues: imaging, gastroenterological, oncological and surgical.

Among the investigational imaging methods, abdominal ultrasound (US) is the first option in the assessment of pancreatic pathology; the sensitivity (Se) of the detection rate in PDAC varies between $70 \%$ and $90 \%$ if the tumor size exceeds $3 \mathrm{~cm}$ [3]. Comparative and complementary to the US are contrast-enhanced contrast ultrasonography (CEUS) that can highlight neoplastic processes with a Se that varies between $86 \%$ and $100 \%$ and abdominal computed tomography (CT) with contrast enhancement showing a diagnostic accuracy between $75 \%-100 \%[4-5]$. 
The shear wave elastography (SWE) technique is congruent with the acoustic radiation force impulse (ARFI) method, allowing acquisition of both qualitative and quantitative data on the elasticity of the parenchymal tissue. In the literature, there is scarce data on the assessment of transabdominal pancreatic stiffness by SWE and ARFI elastographic techniques [6,7].

SWE is based on acoustic shear waves (SW) emitted at the level of a region of interest (ROI); these SW are occurring subsequently to tissue vibrations generated by the transducer through a mechanical excitation [8]. Shear wave data is framed within a function of Young's elastic modulus [9]. Shear Wave Velocity (SWV) values express the stiffness or tissue elasticity at the ROI level in a directly proportional manner [10-13]. SWE computing software has the ability to quantify data in a qualitative (color elastograms) and quantitative manner (SWV expression in $\mathrm{m} / \mathrm{s}$ ), after SW trigger at the level of the ROI. The detected values are analyzed by a calculation algorithm. The transabdominal elastographic method requires further continued validation for diagnosis of pancreatic pathology [14-16]. Endoscopic ultrasonography (EUS) and strain elastography remain an accurate technique in pancreatic tumor evaluation; despite this, it is an invasive method that comes with possible complications due to sedation and endoscopic procedure. The continuous challenge lies at this moment in demonstrating that transabdominal SWE is a reliable technique in the stiffness evaluation of the pancreatic tumors [17-19].

The purpose of this study is to add new relevant data on the stiffness of the pancreatic adenocarcinoma and to evaluate if the transabdominal SWE is a feasible method for differentiation cancer from the normal pancreatic tissue.

\section{Material and method}

The study was a pilot case-control type and was conducted in an observational, analytical, prospective and longitudinal manner. The Ethical Committee approval from University was received and before examination the informed consent of patients was obtained.

\section{Patients}

We included 18 healthy subjects (control group) and 15 patients with pancreatic neoplasms: 14 with pancreatic adenocarcinoma (PDAC group) and one with intraductal papillary mucinous neoplasia (IPMN). Patients were admitted between March and October 2017 in the Gastroenterology departments of the "Octavian Fodor" Regional Institute of Gastroenterology and Hepatology, Cluj Napoca, Romania. Regarding the inclusion criteria for the group of patients with pancreatic neoplasm, the following were selected: a) histopathological material by EUS-FNA (Fine Needle Aspiration) or by surgical procedures and $b$ ) identification of the tumor by $\mathrm{CT}$ and additionally US/CEUS and EUS.

The control group involved subjects without a personal history of pancreatic pathology, absence of chronic alcohol and tobacco consumption and proper pancreas visualization at the US evaluation. Normal pancreas was defined by the absence of sonographically changes encountered in acute pancreatitis (edema, enlargement of the parenchyma), chronic pancreatitis (calcifications, ductal enlargement or presence of cysts) and any focal mass (no matter the aspect: cystic or solid).

Patients were excluded for at least one of the following criteria: non-visualization of the pancreas in the US examination, scarring and/or abdominal tumors located in the pancreas topographic abdominal projection area as well as associated radiotherapy procedures (in the past or at the time of evaluation), gastric tumors, gastro-duodenal stents or gastro-jejunostomy feeding tubes, but also the absence of signing the informed consent. It should be noted that for one patient that required a choledochal stent, the ROI sample was placed strictly in the pancreatic parenchyma and out of contact with the stent.

\section{Ultrasound and elastography evaluation. Outline of the study}

The baseline transabdominal US and SWE evaluation were performed by a single operator, a certified gastroenterologist, using a Logiq E9 (GE Healthcare, Boston, USA) gear with a convex 6 1 transducer (frequency between 1.5 and $6 \mathrm{MHz}$ )

The patients and subjects were examined in the dorsal decubitus position after a fasting period of 12 hours. In order to displace the intestinal bloating and to properly visualize the parenchyma, the patient was asked to avoid deep inspiration breath hold and sometimes short period of apnea were requested during the examination [20]. The abdominal US sections used were transverse or oblique. Following the US assessment of the pancreas and related lesions, a trapezoidal sample was positioned at the parenchyma level outside the contact with digestive tract structures, vessels, hepatic parenchyma, biliary or gastro-duodenal prosthetic materials. Under the conditions of obtaining a proper elastogram in the trapezoidal sample, quantitative measurements were made inside the sample as follows: by placing a circle (ROI) with a diameter of $7.5 \mathrm{~mm}$, which allowed the evaluation of shear wave velocities (SWV) expressed in $\mathrm{m} / \mathrm{s}$ (fig 1).

Mean and median from a minimum number of 5 correctly achieved SWV measurements per segment and le- 


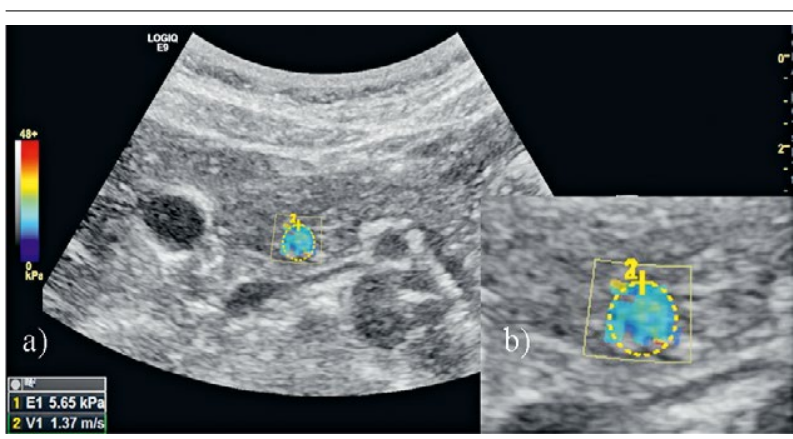

Fig 1. The US-2D view of the cephalic segment, the color elastogram and the adjacent color scale and in the lower left: SWV expressed $\mathrm{m} / \mathrm{s}$ (a) and the color elastogram within the trapezoidal sample with the ROI (b).

sion was considered a target value for obtaining data with statistical relevance. In a previous study we highlighted that there is no significant statistical difference between 5 respective $10 \mathrm{SWV}$ measurements of pancreas elasticity by transabdominal elastography [20]. We recorded for all patients demographic data (age, sex), Wirsung duct diameter, corresponding elastograms and we measured the SWV of the pancreatic parenchyma with adjacent lesions.

\section{Statistical analysis}

Statistical analysis was carried out using the MedCalc Statistical Software version 18 (MedCalc Software bvba, Ostend, Belgium; http://www.medcalc.org; 2018). Quantitative data were tested for the normality of distribution using the Kolmogorov-Smirnov test, and were expressed as mean \pm standard deviation (SD). Differences between groups were assessed by Student $t$ test. The independent influence of age and cancer on the SWE measurements was assessed using the multivariate linear regression. ROC analysis was used in order to estimate the ability of a variable (SWE measurement) to discriminate between two conditions. A cut-off value was chosen where sensitivity (Se) and specificity ( $\mathrm{Sp}$ ) was maximum. A p value $<0.05$ was considered statistically significant.

\section{Results}

\section{Control group}

A total of 18 healthy subjects were included, with an age range between 18 and 40 years $(27.23 \pm 5.06$ years), $60 \%$ female, with an average body mass index (BMI) of $21.49 \pm 3.06 \mathrm{~kg} / \mathrm{m}^{2}$.

Table I expresses the mean values of SWV corresponding to each segment. Correspondingly to the entire parenchyma of the pancreas, the mean SWV was $1.21 \pm 0.27 \mathrm{~m} / \mathrm{s}$.

\section{Pancreatic neoplasm group}

Fifteen patients were included, of which, 14 cases of pancreatic adenocarcinoma and 1 case of intraductal papillary mucinous neoplasia (IPMN). The mean age of patients was $71.38 \pm 14.08$ years, with $69 \%$ being male. Regarding the localization of the tumoral process, all 15 tumors were identified at the cephalic segment.

Table I shows the SWV values from the pancreatic segments corresponding to the tumor site, the area adjacent to the tumoral process and the caudal segment. The mean SWV value at the cephalo-corporeal area including the tumoral site was $1.64 \pm 0.41 \mathrm{~m} / \mathrm{s}$. At the level of the pancreatic body, $75 \%$ of patients presenting a dilated Wirsung duct with a mean diameter of $9.25 \pm 3.82 \mathrm{~mm}$. The mean size of tumor lesions was $30.18 \pm 11.15 \mathrm{~mm}$. The Pearson correlation coefficient of 0.59 , between mean tumor size and mean SWV indicates a moderate positive relationship between those parameters. Table I highlights that although tumors were at the cephalic segment, the mean SWV from the corporeal area proved to be higher than the tumoral SWV and also than the SWV from corporeal measurement in the control group. Corporeal area in the neoplasm group represents the area adjacent to the tumor lesion; no significant differences were found between the head and the body in those with cancer, either between the mean or the median (all $\mathrm{p}>0.05$ ). SWV value of $1.52 \pm 0.19 \mathrm{~m} / \mathrm{s}$ at the caudal level in the pancreatic neoplasm group didn't meet statistical signifi-

Table I. Shear wave velocity values $(\mathrm{m} / \mathrm{s})$ in the neoplasm group versus the control group

\begin{tabular}{|c|c|c|c|c|c|}
\hline \multirow{2}{*}{$\begin{array}{l}\text { Place of } \\
\text { measurement }\end{array}$} & \multirow{2}{*}{$\begin{array}{l}\text { Control group } \\
\text { (18 subjects) }\end{array}$} & \multicolumn{3}{|c|}{ Neoplasm group } & \multirow[t]{2}{*}{ p value } \\
\hline & & $\begin{array}{l}\text { Cephalic tumors } \\
\text { (15 patients) }\end{array}$ & $\begin{array}{l}\text { The area adjacent to the tu- } \\
\text { moral process* (10 patients) }\end{array}$ & $\begin{array}{l}\text { Caudal segment } * * \\
\text { (3 patients) }\end{array}$ & \\
\hline Head - M & $1.27 \pm 0.29$ & $1.49 \pm 0.29$ & & & 0.03 \\
\hline Head $-\mathrm{m}$ & $1.28 \pm 0.31$ & $1.54 \pm 0.32$ & & & 0.02 \\
\hline Body $-M$ & $1.14 \pm 0.23$ & & $1.76 \pm 0.59$ & & 0.01 \\
\hline Body $-m$ & $1.18 \pm 0.25$ & & $1.74 \pm 0.50$ & & 0.01 \\
\hline Tail - M & $1.18 \pm 0.27$ & & & $1.52 \pm 0.19$ & 0.1 \\
\hline Tail $-\mathrm{m}$ & $1.18 \pm 0.25$ & & & $1.54 \pm 0.21$ & 0.07 \\
\hline
\end{tabular}

$\mathrm{M}=$ median; $\mathrm{m}=$ mean; $\mathrm{SWV}=$ Shear wave velocity. * corresponding to the body of the pancreas; $* *$ remnant parenchyma-unaffected by the tumor 


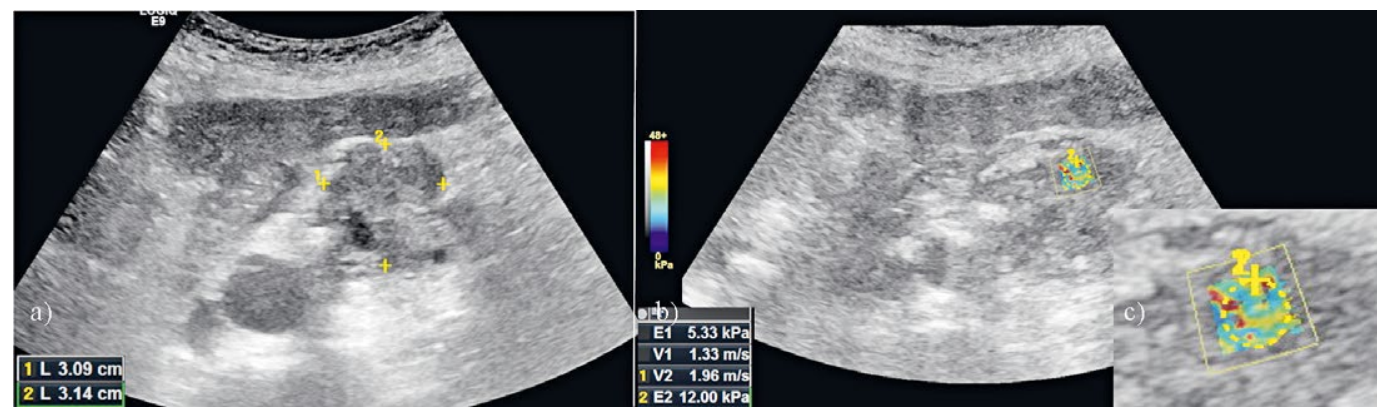

Fig 2. Identification of the pancreatic tumor (a), the color elastogram with SWV expressed in $\mathrm{m} / \mathrm{s}$ (b) and the color elastogram within the trapezoidal sample and the ROI (c) - non-homogeneous pattern of the ROI with red spots inclusions on a background of predominantly green shades.

Table II. The accuracy of the transabdominal Shear Wave Elastography in differentiation of the tumoral lesions from normal parenchyma

\begin{tabular}{llllll}
\hline SWV & AUC & Cut-off $(\mathbf{m} / \mathbf{s})$ & Se $(\mathbf{9 5} \%$ CI) & Sp (95\% CI) & p value \\
\hline Head - M & 0.731 & 1.33 & $81.25(54.4-96.0)$ & $72.22(46.5-90.3)$ & 0.01 \\
Head - m & 0.764 & 1.37 & $81.25(54.4-96.0)$ & $77.78(52.4-93.6)$ & 0.002 \\
Body - M & 0.895 & 1.36 & $77.78(40.0-94.1)$ & $94.12(71.3-99.9)$ & $<0.0001$ \\
Body - m & 0.886 & 1.34 & $77.78(40.0-97.2)$ & $94.12(71.3-99.9)$ & $<0.0001$ \\
\hline
\end{tabular}

$\mathrm{SWV}=$ Shear wave velocity; $\mathrm{M}=$ median; $\mathrm{m}=$ mean; $\mathrm{AUC}=$ area under the curve $\mathrm{CI}=$ confidence interval, $\mathrm{Se}=$ sensibility; $\mathrm{Sp}=\mathrm{specificity}$

cance due to the success rate of the measurements at this level of only $20 \%$. Figure 2 reproduces the non-homogeneous pattern of the elastograms from the tumoral level.

Table II outlines the results regarding the validity of SWE in the differentiation of the tumoral lesions from normal parenchyma, corresponding to the head and corporeal level of the pancreas.

Regarding the Se and specificity (Sp) of the transabdominal SWE in differentiation of tumoral lesions from normal parenchyma, we calculated a cut-off value of $1.33 \mathrm{~m} / \mathrm{s}$ for the head of the pancreas, and $1.36 \mathrm{~m} / \mathrm{s}$ for the body (fig 3 ).

Within the neoplasm group, there was a heterogeneity in terms of age. Table III highlights that this aspect did not influence the SWV values.

In order to find out if cancer is associated with SWE measurements, independently from the influence of age, we used a multivariate linear regression. The presence of cancer was statistically significant associated with SWE mean values for the pancreatic head $(\mathrm{p}=0.02)$ but not with age over 65 years $(p=0.3)$. The patients with cancer had statistically significant higher SWE median values for the pancreatic head $(\mathrm{p}=0.03)$, as the age did not independently influence the stiffness $(p=0.4)$ for this type of pathology. Regarding the adjacent parenchyma of the tumor (corresponding to the body of the pancreas) the results are similar, age over 65 years, did not affect the median values of the $\mathrm{SWV}(\mathrm{p}=0.001)$.
Table III. Independent influence of the tumor on the SWE measurements within the neoplasm group, adjusted for a cutoff of 65 years

\begin{tabular}{|c|c|c|c|}
\hline \multicolumn{3}{|c|}{ SWV (m/s) } & \multirow{2}{*}{ p value } \\
\hline Age (years) & $<65$ & $>65$ & \\
\hline Head - M & $1.35 \pm 0.35$ & $1.43 \pm 0.16$ & 0.3 \\
\hline Head $-\mathrm{m}$ & $1.38 \pm 0.39$ & $1.48 \pm 0.12$ & 0.2 \\
\hline Body $-M$ & $1.30 \pm 0.48$ & $1.60 \pm 0.47$ & 0.2 \\
\hline Body $-\mathrm{m}$ & $1.31 \pm 0.44$ & $1.62 \pm 0.43$ & 0.1 \\
\hline
\end{tabular}

$\mathrm{SWV}=$ Shear wave velocity; $\mathrm{M}=$ median; $\mathrm{m}=$ mean

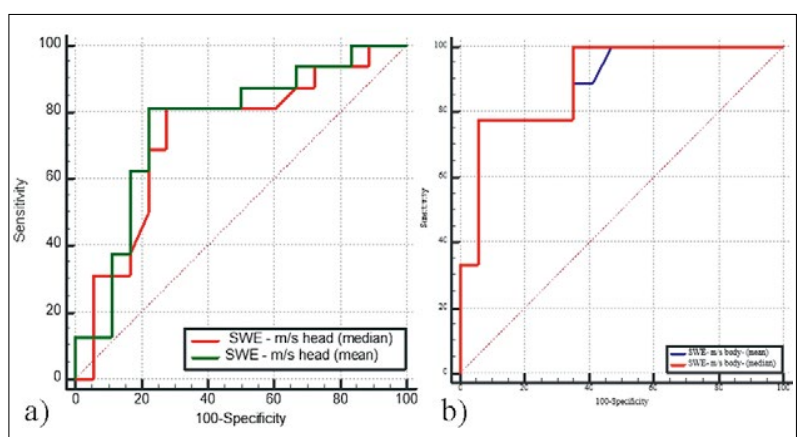

Fig 3. ROC curve regarding the accuracy in distinction the tumoral rigidity from that of a normal parenchyma by transabdominal SWE corresponding to the head (a) and corpus of the pancreas (b). 


\section{Discussions}

Currently, a reduced number of studies regarding SWV values by transabdominal elastography for pancreatic tumor lesions were published. Park et al found an average SWV in pancreatic tumors of $3.3 \pm 1.0 \mathrm{~m} / \mathrm{s}(1.3-4.8)$ and at the adjacent parenchyma of $1.55 \pm 0.6 \mathrm{~m} / \mathrm{s}[18]$ and Kawada et al showed a mean SWV of adjacent neoplasia of $1.51 \pm 0.45 \mathrm{~m} / \mathrm{s}$, the logistics used by the two authors being quasi-identical, namely a Siemens Acuson S2000 (Siemens, Mountain View, USA) [18,21-22].

Compared to the ARFI (with Virtual Touch Quantification - VTQ software) exploration, SWE- Logiq E9 analysis also allows a qualitative evaluation by expressing a color elastogram associated with exposed values. SWV mean values through ARFI and SWE for normal pancreatic parenchyma are similar [20,23].

We obtained statistically significant higher SWE values at the tumor site versus the values found in the case of the normal pancreas $(1.54 \pm 0.32$ vs $1.21 \pm 0.27 \mathrm{~m} / \mathrm{s})$. Because some of the tumors had a poorly defined extension at the US evaluation, we also highlighted a mean SWV for the cephalo-corporeal area including the tumoral site of $1.64 \pm 0.41 \mathrm{~m} / \mathrm{s}$.

The low success rate of the measurements at the caudal level is explained by the non-visualization of a proper ROI to provide a qualitatively accurate assessment.

The mean SWV value obtained at the body of the pancreas, adjacent to tumoral processes, but nonetheless non-tumorous parenchyma, was $1.74 \pm 0.50 \mathrm{~m} / \mathrm{s}$, similar to that obtained by Kawada et al [21]. SWV was evaluated corresponding to the remnant corporeal parenchyma and anterior from the dilated Wirsung duct. In our opinion, pathological aspects due to upstream obstruction (such as chronic inflammation) could influence morphological changes and increase the parenchymal stiffness. This aspect should be considered in the interpretation of the "residual" pancreatic tissue, in a proper clinical setting.

Due to a wide-range of age in the neoplasm group ( $71.38 \pm 14.08$ years) we tried to prefigure a possible, although an improbable effect on the values of SWV. We used a cut-off for age of 65 years, according to the World Health Organization definition of elderly and adults [24]. Even if the SWV values for the elderly subjects were slightly increased, we proved that it is not statistically relevant. We chose a control group under the age of 65 due to the existence of clear cut-off values for normal pancreas and to eliminate biased results due to possible morphological changes that can occur in the age range of 50 and 65 years. We demonstrated the independent influence of the tumor on the SWE measurements, adjusted for age.
Most of the data regarding the elastographic evaluation of the pancreas come from studies of endoscopic ultrasonography (EUS) with strain elastography; this includes qualitative (elastograms) and quantitative assessment. Since 2005, EUS elastography has been used for the differential diagnosis between solid malignant and benign tumors [25-26]. Despite the fact that the differentiation benchmark between benign and malignant pancreatic lesions is not totally homogenous in the literature, EUS elastography achieves a range of Se from $95 \%$ to $99 \%$, respectively a Sp between $67 \%$ to $76 \%$ [27-28].

The group of quantitative methods of diagnosis for pancreatic tumors includes among EUS-strain elastography and transabdominal SWE, also neural network analysis and strain histogram [29]. Neural network analysis was developed by Saftoiu et al and is accomplished by computer software [6,30-31].

Regarding the aspect of the elastograms from EUSstrain elastography, the homogenous pattern and the dominant color is taken into consideration. A completely green appearance is suggestive for a normal parenchyma, while a blue color indicates a possible neuroendocrine tumor; green spots on a blue background indicates a pancreatic ductal adenocarcinoma, while a green background with blue and red inclusions suggests chronic pancreatitis [6].

Also, in our study, the appearance between the elastograms of the control and the neoplasm group differs by color and pattern. In the case of the normal pancreas, the predominant color is blue and the pattern type is a homogeneous one; while in the case of the neoplasia group the elastograms identified are quite heterogeneously. A specific pattern for tumoral lesions could not be established.

A technical element still difficult to overcome is the relatively low depth of the ROI $(6-8 \mathrm{~cm})$, which justifies the relative low success rate in SWV determination at a caudal level for those within the neoplasm group. The absence of sufficient leftover parenchyma to allow the ROI sample to retrieve SWV data should also be taken into consideration.

Among the limitations in the present study we present: a) the small size group of the patients, b) age matched control between groups was not entirely accomplished but we pointed out that this was not statistically significant and c) the lack of an inter- and intraobserver agreement.

\section{Conclusions}

Our study results confirm statistically significant higher SWV at the tumor site $(1.54 \pm 0.32 \mathrm{~m} / \mathrm{s})$ versus the normal pancreas. There is a positive correlation between 
the SWV and tumor size. At the caudal level, we encountered a low success rate of the SWV measurements, due to improper ROI visualization. SWV from the remnant corporeal parenchyma, the area adjacent to the neoplasia, were elevated; we interpreted an increased stiffness by morphological changes due to the enclosed obstruction. In conclusion, transabdominal SWE represents a surrogate but feasible method for differentiation of cancer from the normal pancreatic tissue.

\section{Conflict of interest: none}

Acknowledgements: We thank assistant Zsoka Szasz for all her support during the evaluation of the patients.

\section{References}

1. D'Onofrio M, Canestrini S, Crosara S, Robertis RD, Mucelli RP. Contrast enhanced ultrasound with quantitative perfusion analysis for objective characterization of pancreatic ductal adenocarcinoma: A feasibility study. World J Radiol 2014;6: 31-35.

2. Porta M, Fabregat X, Malats N, et al. Exocrine pancreatic cancer: symptoms at presentations and their relation to tumour site and stage. Clin Transl Oncol 2005;7:189-197.

3. Tanaka S, Kitamra T, Yamamoto K, et al. Evaluation of routine sonography for early detection of pancreatic cancer. Jpn J Clin Oncol 1996;26:422-427.

4. D’Onofrio M, Crosara S, Signorini M, et al. Comparasion between CT and CEUS in the diagnosis of pancreatic adenocarcinoma. Ultraschall Med 2013;34:377-381.

5. Dimcevski G, Erchinger FG, Havre R, Gilja OH. Ultrasonography in diagnosing chronic pancreatitis: new aspects. World J Gastroenterol 2013;19:7247-7257.

6. Kawada N, Tanaka S. Elastography for the pancreas: Current status and future perspective. World J Gastroenterol 2016;22:3712-3724.

7. Hirooka Y, Kuwahara T, Irisawa A, et al. JSUM ultrasound elastography practice guidelines: pancreas. J Med Ultrason (2001) 2015;42:151-174.

8. Bamber J, Cosgrove D, Dietrich CF, et al. EFSUMB guidelines and recommendations on the clinical use of ultrasound elastography. Part 1: Basic principles and technology. U1traschall Med 2013;34:169-184.

9. McAleavey SA, Menon M, Orszulak J. Shear-modulus estimation by application of spatially-modulated impulsive acoustic radiation force. Ultrason Imaging 2007;29:87-104.

10. Nightingale KR, Bentley R, Trahey G. Observations of tissue response to acoustic radiation force: opportunities for imaging. Ultrason Imaging 2002;24:129-138.

11. Nightingale K, McAleavey S, Trahey G. Shear-wave generation using acoustic radiation force: in vivo and ex vivo results. Ultrasound Med Biol 2003;29:1715-1723.

12. Castéra L, Vergniol J, Foucher J, et al. Prospective comparison of transient elastography, Fibrotest, APRI, and liver biopsy for the assessment of fibrosis in chronic hepatitis $\mathrm{C}$. Gastroenterology 2005;128:343-350.

13. Shiina T, Nightingale KR, Palmeri ML, et al. WFUMB guidelines and recommendations for clinical use of ultrasound elastography: Part 1: basic principles and terminology. Ultrasound Med Biol 2015;41:1126-1147.

14. Nightingale K, Soo MS, Nightingale R, Trahey G. Acoustic radiation force impulse imaging: in vivo demonstration of clinical feasibility. Ultrasound Med Biol 2002;28:227-235.

15. McAleavey SA, Menon M, Orszulak J. Shear-modulus estimation by application of spatially-modulated impulsive acoustic radiation force. Ultrason Imaging 2007;29:87-104.

16. Nightingale K, Bentley R, Trahey G. Observations of tissue response to acoustic radiation force: opportunities for imaging. Ultrason Imaging 2002;24:129-138.

17. D’Onofrio M, Gallotti A, Martone E, Pozzi Mucelli R. Solid appearance of pancreatic serous cystadenoma diagnosed as cystic at ultrasound acoustic radiation force impulse imaging. JOP 2009;10:543-546.

18. Park MK, Jo J, Kwon H, et al. Usefulness of acoustic radiation force impulse elastography in the differential diagnosis of benign and malignant solid pancreatic lesions. Ultrasonography 2014;33:26-33.

19. Harada N, Ishizawa T, Inoue $Y$, et al. Acoustic radiation force impulse imaging of the pancreas for estimation of pathologic fibrosis and risk of postoperative pancreatic fistula. J Am Coll Surg 2014;219:887-894.

20. Zaro R, Lupsor-Platon M, Cheviet A, Badea R. The pursuit of normal reference values of the pancreatic stiffness by using Acoustic Radiation Force Impulse (ARFI) elastography. Med Ultrason 2016;18:425-430.

21. Kawada N, Tanaka S, Uehara H, et al. Potential use of point shear wave elastography for the pancreas: A single-center prospective study. Eur J Radiol 2014;83:620-624.

22. Yashima $Y$, Sasahira N, Isayama $H$, et al. Acoustic radiation force impulse elastography for noninvasive assessment of chronic pancreatitis. J Gastroenterol 2012;47:427-432.

23. Mateen MA, Muheet KA, Mohan RJ, et al. Evaluation of ultrasound based acoustic radiation force impulse (ARFI) and eSie touch sonoelastography for diagnosis of inflammatory pancreatic diseases. JOP 2012;13:36-44.

24. Chantarojanasiri T, Hirooka Y, Kawashima H, et al. Agerelated changes in pancreatic elasticity: When should we be concerned about their effect on strain elastography? Ultrasonics 2016;69:90-96.

25. Mei M, Ni J, Liu D, Jin P, Sun L. EUS elastography for diagnosis of solid pancreatic masses: a meta-analysis. Gastrointest Endosc 2013;77:578-589.

26. Li X, Xu W, Shi J, Lin Y, Zeng X. Endoscopic ultrasound elastography for differentiating between pancreatic adenocarcinoma and inflammatory masses: a meta-analysis. World J Gastroenterol 2013;19:6284-6291.

27. Iglesias-Garcia J, Larino-Noia J, Abdulkader I, Forteza J, Dominguez-Munoz JE. Quantitative endoscopic ultrasound elastography: an accurate method for the differentiation of solid pancreatic masses. Gastroenterology 2010;139:11721180 . 
28. Iglesias-Garcia J, Larino-Noia J, Abdulkader I, Forteza J, Dominguez-Munoz JE. EUS elastography for the characterization of solid pancreatic masses. Gastrointest Endosc 2009;70:1101-1108.

29. Săftoiu A, Vilmann P. Differential diagnosis of focal pancreatic masses by semiquantitative EUS elastography: between strain ratios and strain histograms. Gastrointest Endosc 2013;78:188-189.
30. Săftoiu A, Vilmann P, Gorunescu F, et al. Efficacy of an artificial neural network-based approach to endoscopic ultrasound elastography in diagnosis of focal pancreatic masses. Clin Gastroenterol Hepatol 2012;10:84-90.

31. Săftoiu A, Vilmann P, Gorunescu F, et al. Neural network analysis of dynamic sequences of EUS elastography used for the differential diagnosis of chronic pancreatitis and pancreatic cancer. Gastrointest Endosc 2008;68:1086-1094. 